\title{
FRACTIONAL POWERS OF OPERATORS, $K$-FUNCTIONALS, ULYANOV INEQUALITIES
}

\author{
WALTER TREBELS \\ AG 5, Fb. Mathematik, Technische Universität Darmstadt \\ Schlossgartenstr. 7, 64289 Darmstadt, Germany \\ E-mail: trebels@mathematik.tu-darmstadt.de \\ URSULA WESTPHAL \\ Institut für Analysis, Leibniz Universität Hannover \\ Welfengarten 1, 30167 Hannover, Germany \\ E-mail: westphal@math.uni-hannover.de
}

\begin{abstract}
Given an equibounded $\left(\mathcal{C}_{0}\right)$-semigroup of linear operators with generator $A$ on a Banach space $X$, a functional calculus, due to L. Schwartz, is briefly sketched to explain fractional powers of $A$. Then the (modified) $K$-functional with respect to $\left(X, D\left((-A)^{\alpha}\right)\right), \alpha>0$, is characterized via the associated resolvent $R(\lambda ; A)$. Under the assumption that the resolvent satisfies a Nikolskii type inequality, $\|\lambda R(\lambda ; A) f\|_{Y} \leq c \varphi(1 / \lambda)\|f\|_{X}$, for a suitable Banach space $Y$, an Ulyanov inequality is derived. This will be of interest if one has good control on the resolvent but not on the semigroup.
\end{abstract}

1. Introduction. Let $\left(X,\|\cdot\|_{X}\right)$ be a Banach space, $\{T(t): t \geq 0\}$ be an equibounded semigroup of class $\left(\mathcal{C}_{0}\right)$ of linear operators from $X$ into itself, that is,

$$
\begin{aligned}
T\left(t_{1}\right) T\left(t_{2}\right) & =T\left(t_{1}+t_{2}\right) \quad \text { for } t_{1}, t_{2} \geq 0, \quad T(0)=I, \\
\lim _{t \rightarrow 0+}\|T(t) f-f\|_{X} & =0 \text { for each } f \in X \quad\left(\left(\mathcal{C}_{0}\right) \text {-property }\right) . \\
\|T(t)\|_{\mathcal{L}(X)} & \leq M \text { for all } t \geq 0, M \text { being a constant. }
\end{aligned}
$$

The infinitesimal generator $A$ of $\{T(t): t \geq 0\}$ is a closed linear operator defined by

$$
\text { Af }:=\lim _{t \rightarrow 0+} t^{-1}[T(t) f-f]
$$

2000 Mathematics Subject Classification: Primary 47D60, 46B70; Secondary 41A17, 41A65.

Key words and phrases: $K$-functionals, semigroups of operators, resolvents, L. Schwartz functional calculus, Ulyanov inequality, Nikolskii inequality.

The paper is in final form and no version of it will be published elsewhere. 
for all $f \in X$ for which this limit exists in the norm topology of $X$. The set of all these elements $f \in X$ is the domain $D(A)$ of $A$; it is dense in $X$. For positive real $\alpha$ we introduce the fractional power operator $(-A)^{\alpha}$ of $A$ with domain $D\left((-A)^{\alpha}\right)$ by a functional calculus due to L. Schwartz [6] (see Section 2) and consider the (modified) $K$-functional for the couple $\left(X, D\left((-A)^{\alpha}\right)\right)$

$$
K\left(t^{\alpha}, f ; X, D\left((-A)^{\alpha}\right)\right):=\inf _{g \in D\left((-A)^{\alpha}\right)}\left\{\|f-g\|+t^{\alpha}\left\|(-A)^{\alpha} g\right\|\right\} .
$$

As is well-known, $K$-functionals give rise to a real interpolation method, due to J. Peetre [5]. In connection with embedding theorems it may be interesting to relate $K$-functionals on $X$ to corresponding ones on another Banach space $Y$.

The aim of this paper is to give some results in this direction which continue former investigations in a recent paper [8]. To be more precise, let us denote for a moment the initial semigroup on $X$ by $\left\{T_{X}(t): t \geq 0\right\}$ and its infinitesimal generator by $A_{X}$. Assume that $\left(Y,\|\cdot\|_{Y}\right)$ is a further Banach space which is compatible with $X$, i.e., $X$ and $Y$ are continuously embedded in some Hausdorff topological vector space. Let $A_{Y}$ denote the infinitesimal generator of an equibounded $\left(\mathcal{C}_{0}\right)$-semigroup $\left\{T_{Y}(t): t \geq 0\right\}$ on $Y$, which is consistent with the given semigroup on $\left(X,\|\cdot\|_{X}\right)$, i.e., $T_{X}(t) f=T_{Y}(t) f$ if $f \in X \cap Y$. If there is no confusion we drop the subscripts $X$ and $Y$ in the notation of the semigroups and their infinitesimal generators, and under the assumptions just described we say that $\{T(t): t \geq 0\}$ is a consistent semigroup on the compatible pair $(X, Y)$ of Banach spaces.

If $\{T(t): t \geq 0\}$ is such a semigroup let us assume, in addition, that for each positive $t$ the operator $T(t)$ maps $X$ continuously into $Y$ and satisfies some Nikolskii type condition

$\left(\mathrm{N}_{\varphi}\right) \quad\|T(t) T(t) f\|_{Y} \leq c \varphi(t)\|T(t) f\|_{X} \quad$ for all $f \in X, t>0$,

where $c>0$ is independent of $f \in X$ and $t>0$, and $\varphi:(0, \infty) \rightarrow(0, \infty)$ is an essentially decreasing function, i.e., $\varphi(s) \leq c \varphi(t)$ for all $s>t>0$.

Under these assumptions the following theorem was proved in [8].

TheOREM A. If for some $f \in X$ and some $\alpha>0$

$$
\int_{0}^{t} \varphi(u) K\left(u^{\alpha}, f ; X, D_{X}\left((-A)^{\alpha}\right)\right) \frac{d u}{u}<\infty, \quad t>0,
$$

then $f \in Y$ and the following Ulyanov type inequality holds

$$
K\left(t^{\alpha}, f ; Y, D_{Y}\left((-A)^{\alpha}\right)\right) \leq c \int_{0}^{t} \varphi(u) K\left(u^{\alpha}, f ; X, D_{X}\left((-A)^{\alpha}\right)\right) \frac{d u}{u} .
$$

Concerning applications we often have the situation that an explicit representation of the semigroup generated by some closed linear operator is not available. Having the theorem of Hille-Yosida in mind, it might be convenient in these cases to replace the Nikolskii type condition $\left(N_{\varphi}\right)$ by an estimate involving the resolvent of the infinitesimal generator instead of the semigroup.

Condition $\left(\mathrm{N}_{\varphi, \lambda_{0}}^{*}\right)$. Let $\{T(t): t \geq 0\}$ be a consistent semigroup with generator $A$ on a compatible pair $(X, Y)$ of Banach spaces. Let $\varphi:(0, \infty) \rightarrow(0, \infty)$ be an essentially decreasing function and $\lambda_{0} \geq 0$. We say that $\{T(t)\}$ satisfies a Nikolskii type condition 
$\left(\mathrm{N}_{\varphi, \lambda_{0}}^{*}\right)$ with respect to $(X, Y)$, if for each $\lambda>\lambda_{0}$ the resolvent operator $R(\lambda ; A):=$ $(\lambda I-A)^{-1}$ maps $X$ continuously into $Y$ such that

$$
\|\lambda R(\lambda ; A) f\|_{Y} \leq c \varphi\left(\lambda^{-1}\right)\|f\|_{X}, \quad f \in X, \lambda>\lambda_{0} .
$$

If we consider Ulyanov type inequalities for integer $\alpha$, the underlying semigroup need not be equibounded.

EXAMPLE 1.1. Let $\Omega \subset \mathbf{R}^{n}$ be an open and bounded set with a uniformly $C^{2}$-boundary $\partial \Omega$ - see [3, p. 2]. Consider a uniformly elliptic [3, p. 71] second order differential operator

$$
\mathcal{A}(x, D)=\sum_{i, j=1}^{n} a_{i j}(x) D_{i j}+\sum_{i=1}^{n} b_{i}(x) D_{i}+c(x) I
$$

with real, uniformly continuous and bounded coefficients $a_{i j}, b_{i}, c$. Let $X=L^{p}(\Omega), 1<$ $p<\infty$, and set

$$
D\left(A_{p}\right)=W^{2, p}(\Omega) \cap W_{0}^{1, p}(\Omega), \quad A_{p} u=\mathcal{A}(\cdot, D) u \quad \text { for } u \in D\left(A_{p}\right) ;
$$

here $W^{2, p}(\Omega)$ is the standard second order Sobolev space on $L^{p}(\Omega), W_{0}^{1, p}(\Omega)$ the completion (with respect to the first order Sobolev norm) of the $C^{\infty}$-functions with compact support in $\Omega$. Then $A_{p}$ is a closed linear operator, densely defined in $L^{p}(\Omega)$. By [3, p. 73], there exist $\omega_{p} \in \mathbf{R}$ and $M_{p}>0$ such that $\left\{\lambda \in \mathbf{C}: \operatorname{Re} \lambda \geq \omega_{p}\right\} \subset \rho\left(A_{p}\right)$ and

$$
|\lambda|\|u\|_{p} \leq M_{p}\left\|\lambda u-A_{p} u\right\|_{p} \quad \operatorname{Re} \lambda \geq \omega_{p}, \quad u \in D\left(A_{p}\right) .
$$

Thus, $A_{p}$ is a sectorial operator and generates a holomorphic semigroup of class $\left(\mathcal{C}_{0}\right)$ on $L^{p}(\Omega)$. From now on we assume that $p>n$. Then, by $[1$, p. 98, p. 108]

$$
W^{2, p}(\Omega) \hookrightarrow C^{1}(\bar{\Omega}), \quad W^{2, p}(\Omega) \hookrightarrow L^{q}(\Omega), \quad p \leq q \leq \infty .
$$

Here $C^{1}(\bar{\Omega})$ is the subset of those elements of $C^{1}(\Omega)$ which are bounded and uniformly continuous on $\Omega$ together with their first partial derivatives. Now the following Nikolskii inequality holds (replace $u$ by $R\left(\lambda ; A_{p}\right) u$ ):

If $n<p<q \leq \infty$, then there exists a $\lambda_{p}>0$ such that

$$
\|\lambda u\|_{q} \leq c \lambda^{\frac{n}{2}(1 / p-1 / q)}\left\|\lambda u-A_{p} u\right\|_{p}, \quad \lambda \geq \lambda_{p} .
$$

For $q=\infty$ this is just Theorem 3.1.19 in [3] (observe that we may choose there $g_{0}=0$ ); in the case $q<\infty$ note $|u(x)|^{q}=|u(x)|^{q-p}|u(x)|^{p}$, apply upon the first factor the $(q=\infty)$-estimate, whereas for the second factor observe that $\|\lambda u\|_{p} \leq c\left\|\lambda u-A_{p} u\right\|_{p}$.

THEOREM 1.2. Let $\{T(t): t \geq 0\}$ be a consistent semigroup on a compatible pair $(X, Y)$ of Banach spaces satisfying a Nikolskii type condition $\left(\mathrm{N}_{\varphi, \lambda_{0}}^{*}\right)$. If for some $f \in X$ and $\alpha>0$

$$
\int_{0}^{t} \varphi(u) K\left(u^{\alpha}, f ; X, D_{X}\left((-A)^{\alpha}\right)\right) \frac{d u}{u}<\infty, \quad 0<t<\lambda_{0}^{-1},
$$

then $f \in Y$ and for all $\lambda>\lambda_{0}$

$$
K\left(\lambda^{-\alpha}, f ; Y, D_{Y}\left((-A)^{\alpha}\right)\right) \leq c \int_{0}^{1 / \lambda} \varphi(u) K\left(u^{\alpha}, f ; X, D_{X}\left((-A)^{\alpha}\right)\right) \frac{d u}{u} .
$$

For $\alpha \in \mathbf{N}$ the assumption of the equiboundedness of the semigroup can be omitted. 
Let us rewrite Theorem 1.2 in terms of generalized moduli of continuity. To this end consider the fractional difference operator $[I-T(s)]^{\alpha}, \alpha>0$,

$$
[I-T(s)]^{\alpha}=\sum_{j=0}^{\infty}(-1)^{j}\left(\begin{array}{c}
\alpha \\
j
\end{array}\right) T(j s), \quad\left(\begin{array}{l}
\beta \\
j
\end{array}\right)=\prod_{k=1}^{j} \frac{\beta-k+1}{k}, \quad \beta \in \mathbf{R} .
$$

Now we observe that the associated modulus of continuity

$$
\omega_{\alpha}(t, f ;\{T(\cdot)\})_{X}:=\sup _{0<s<t}\left\|[I-T(s)]^{\alpha} f\right\|_{X}
$$

gives a characterization of the $K$-functional (see [7, Theorem 1.1]),

$$
\omega_{\alpha}(t, f ;\{T(\cdot)\})_{X} \approx K\left(t^{\alpha}, f ; X, D_{X}\left((-A)^{\alpha}\right)\right),
$$

and thus we arrive at the following result.

Corollary 1.3. Under the hypotheses of Theorem 1.2,

$$
\omega_{\alpha}(t, f ;\{T(\cdot)\})_{Y} \leq c \int_{0}^{t} \varphi(u) \omega_{\alpha}(u, f ;\{T(\cdot)\})_{X} \frac{d u}{u} .
$$

Our proof of Theorem 1.2 depends on the following characterization of the $K$-functional via the resolvent of the generator $A$.

TheOREM 1.4. Let $\{T(t): t \geq 0\}$ be an equibounded $\left(\mathcal{C}_{0}\right)$-semigroup of operators on a Banach space $X$ with infinitesimal generator $A$. Then, for all $f \in X, \lambda>0$, and $\alpha>0$,

$$
K\left(\lambda^{-\alpha}, f ; X, D\left((-A)^{\alpha}\right)\right) \approx\left\|[I-\lambda R(\lambda ; A)]^{\alpha} f\right\| .
$$

2. Functional calculus. To explain fractional powers of operators associated with the infinitesimal generator of an equibounded $\left(\mathcal{C}_{0}\right)$-semigroup we use a functional calculus due to Laurent Schwartz [6] (see also [9]) which is an elegant and powerful tool for our purposes. Concerning an approach to fractional powers of operators in the framework of the more general class of non-negative operators we refer, e.g., to the book of Martinez and Sanz [4].

Let $X$ be a Banach space and $\mathcal{L}(X)$ be the Banach algebra of endomorphisms of $X$. Let $\{T(t): t \geq 0\}$ be an equibounded $\left(\mathcal{C}_{0}\right)$-semigroup of operators in $\mathcal{L}(X)$ with infinitesimal generator $A$. Denote by $\mathcal{M}\left(\mathbf{R}_{+}\right)$the space of finite Borel measures on the interval $[0, \infty)$. For $\mu \in \mathcal{M}\left(\mathbf{R}_{+}\right)$consider the Laplace transform of $\mu$,

$$
\widehat{\mu}(z):=\int_{0}^{\infty} e^{-z t} d \mu(t), \quad \operatorname{Re} z>0 .
$$

Then the formal correspondence $e^{-z t} \sim T(t)=e^{t A}$ gives rise to the definition of an operator $G(\mu) \in \mathcal{L}(X)$

$$
G(\mu) f:=\int_{0}^{\infty} T(t) f d \mu(t), \quad f \in X .
$$

If $\mu$ has a density with respect to the Lebesgue measure, say $d \mu(t)=h(t) d t$ with $h \in L^{1}(0, \infty)$, then we set

$$
G(h) f:=G(\mu) f=\int_{0}^{\infty} h(t) T(t) f d t, \quad f \in X .
$$


For instance, the Dirac measure $\delta_{t}$ at $t \geq 0$ yields the semigroup operator $G\left(\delta_{t}\right)=T(t)$, while the resolvent of $A$ is given by

$$
G\left(e_{\lambda} \chi_{(0, \infty)}\right) f=\int_{0}^{\infty} e^{-\lambda t} T(t) f d t=R(\lambda ; A) f, \quad f \in X,
$$

where $e_{\lambda}$ denotes the exponential function $e_{\lambda}(t):=e^{-\lambda t}, \lambda>0, t \in \mathbf{R}$, and $\chi_{(0, \infty)}$ is the characteristic function of the interval $(0, \infty)$.

The mapping $\mu \mapsto G(\mu)$ is an algebra homomorphism of $\mathcal{M}\left(\mathbf{R}_{+}\right)$into $\mathcal{L}(X)$. It can be extended to a class of integrable distributions in the following way.

Let $\mathcal{D}_{L^{1}}^{\prime}\left(\mathbf{R}_{+}\right)$be the class of distributions on $\mathbf{R}$ which are finite sums of derivatives of finite Borel measures and whose supports are contained in the interval $[0, \infty)$. Consider the filter $\mathcal{F}$ on $\mathcal{D}(\mathbf{R})$ with base $\left\{F_{\varepsilon}: \varepsilon>0\right\}$, where

$$
F_{\varepsilon}:=\left\{\varphi \in \mathcal{D}(\mathbf{R}): \varphi \geq 0, \operatorname{supp} \varphi \subset[0, \varepsilon),\left|\int_{0}^{\infty} \varphi(t) d t-1\right|<\varepsilon\right\} .
$$

This filter converges to the Dirac measure $\delta$ with respect to the topology of $\mathcal{D}^{\prime}(\mathbf{R})$. If $U \in \mathcal{D}_{L^{1}}^{\prime}\left(\mathbf{R}_{+}\right)$and $\varphi \in \mathcal{D}(\mathbf{R})$ with $\operatorname{supp} \varphi \subset[0, \infty)$, then the convolution of $U$ and $\varphi, U * \varphi$, belongs to $L^{1}(0, \infty)$. Thus the operator $G(U)$ is defined by the formula

$$
G(U) f:=\lim _{\mathcal{F}} G(U * \varphi) f=\lim _{\mathcal{F}} \int_{0}^{\infty}(U * \varphi)(t) T(t) f d t,
$$

where the limit is taken with respect to the norm topology of $X$, as $\varphi$ approaches $\delta$ following the filter $\mathcal{F}$. The domain $D(G(U))$ is the set of all $f \in X$ for which the limit exists. $G(U)$ is a closed linear operator and its domain is dense in $X$.

The most effective tool of the functional calculus $G$ associated with the semigroup $\{T(t): t \geq 0\}$ is the Convolution Theorem.

Theorem 2.1 (Convolution). Let $U, V \in \mathcal{D}_{L^{1}}^{\prime}\left(\mathbf{R}_{+}\right)$and $f \in D(G(V))$. Then $U * V \in$ $\mathcal{D}_{L^{1}}^{\prime}\left(\mathbf{R}_{+}\right)$, and $f \in D(G(U * V))$ if and only if $G(V) f \in D(G(U))$. In this case

$$
G(U * V) f=G(U) G(V) f .
$$

One easily verifies that

$$
G\left(\delta^{n}\right)=(-A)^{n}, \quad n \in \mathbf{N},
$$

where $\delta^{n}$ denotes the $n$-th derivative of the Dirac measure $\delta$. More generally, fractional powers $(-A)^{\alpha}$ for non-integer positive $\alpha$ may be defined with the aid of fractional derivatives of $\delta$. The family $\left\{\delta^{\alpha}: \alpha \in \mathbf{C}\right\}$ is an analytic family of tempered distributions on $\mathbf{R}$ whose supports are contained in $[0, \infty)$ and which have the Laplace transforms

$$
\widehat{\delta}^{\alpha}(z)=z^{\alpha}, \quad \operatorname{Re} z>0 .
$$

We are interested only in the real $\alpha$ 's. Thus, $\delta^{0}:=\delta$, and for $\alpha>0$ we have the representations

$$
\begin{gathered}
\left\langle\varphi, \delta^{-\alpha}\right\rangle=\frac{1}{\Gamma(\alpha)} \int_{0}^{\infty} u^{\alpha-1} \varphi(u) d u, \quad \varphi \in \mathcal{D}(\mathbf{R}), \\
\left\langle\varphi, \delta^{\alpha}\right\rangle=\frac{(-1)^{m}}{\Gamma(m-\alpha)} \int_{0}^{\infty} u^{m-\alpha-1} \varphi^{(m)}(u) d u, \quad 0<\alpha<m, m \in \mathbf{N}, \varphi \in \mathcal{D}(\mathbf{R}) .
\end{gathered}
$$


As $\delta^{\alpha} \in \mathcal{D}_{L^{1}}^{\prime}\left(\mathbf{R}_{+}\right)$for $\alpha>0$, we define

$$
(-A)^{\alpha}:=G\left(\delta^{\alpha}\right), \quad \alpha>0 .
$$

By the convolution theorem,

$$
(-A)^{\alpha}(-A)^{\gamma}=(-A)^{\alpha+\gamma}, \quad \alpha, \gamma>0 .
$$

The operator $A-\lambda I, \lambda>0$ generates the equibounded $\left(\mathcal{C}_{0}\right)$-semigroup $\left\{e_{\lambda}(t) T(t)\right.$ : $t \geq 0\}$, thus the fractional powers $(\lambda I-A)^{\alpha}, \alpha>0$, are well-defined by the functional calculus associated with this semigroup. One easily shows that

$$
(\lambda I-A)^{\alpha}=G\left(e_{\lambda} \delta^{\alpha}\right), \quad \alpha, \lambda>0,
$$

and $D\left((\lambda I-A)^{\alpha}\right)=D\left((-A)^{\alpha}\right)$ for all $\lambda>0$. As $e_{\lambda} \delta^{-\alpha}, \alpha>0$, is a regular distribution generated by an $L^{1}(0, \infty)$-function and satisfying

$$
e_{\lambda} \delta^{-\alpha} * e_{\lambda} \delta^{\alpha}=\delta
$$

the operator $G\left(e_{\lambda} \delta^{-\alpha}\right)$ is the bounded inverse of $(\lambda I-A)^{\alpha}$. Note that $G\left(e_{\lambda} \delta^{-\alpha}\right)$ is just the resolvent of $A$ if $\alpha=1$. Thus we set

$$
R^{\alpha}(\lambda ; A) f:=G\left(e_{\lambda} \delta^{-\alpha} f\right)=\frac{1}{\Gamma(\alpha)} \int_{0}^{\infty} t^{\alpha-1} e^{-\lambda t} T(t) f d t, \quad \alpha, \lambda>0, f \in X .
$$

We collect some properties of the operators $R^{\alpha}(\lambda ; A)$ which are needed in the sequel. Obviously,

$$
\begin{gathered}
\left\|\lambda^{\alpha} R^{\alpha}(\lambda ; A)\right\| \leq M, \quad \alpha, \lambda>0, \\
R^{\alpha}(\lambda ; A) R^{\gamma}(\lambda ; A)=R^{\alpha+\gamma}(\lambda ; A), \quad \alpha, \gamma, \lambda>0 .
\end{gathered}
$$

Moreover, for $0 \leq \lambda \leq \sigma, \sigma>0$ fixed, we have

$$
e_{\lambda} \delta^{\alpha} * e_{\sigma} \delta^{-\alpha}=\sum_{j=0}^{\infty}(-1)^{j}\left(\begin{array}{c}
\alpha \\
j
\end{array}\right)(\sigma-\lambda)^{j} e_{\sigma} \delta^{-j}=: \rho_{\lambda, \sigma}^{\alpha},
$$

where the right hand side defines a finite Borel measure $\rho_{\lambda, \sigma}^{\alpha}$ on $[0, \infty)$. Thus, $G\left(\rho_{\lambda, \sigma}^{\alpha}\right) \in$ $\mathcal{L}(X)$ and

$$
G\left(\rho_{\lambda, \sigma}^{\alpha}\right)=\sum_{j=0}^{\infty}(-1)^{j}\left(\begin{array}{c}
\alpha \\
j
\end{array}\right)(\sigma-\lambda)^{j} R^{j}(\sigma ; A) .
$$

Since $(\sigma-\lambda) R(\sigma ; A)-I$ generates an equibounded (even uniformly continuous) semigroup $\{S(t): t \geq 0\}$,

$$
S(t):=e^{-t} \sum_{j=0}^{\infty} \frac{t^{j}(\sigma-\lambda)^{j}}{j !} R^{j}(\sigma ; A), \quad t>0
$$

the representation

$$
G\left(\rho_{\lambda, \sigma}^{\alpha}\right)=[I-(\sigma-\lambda) R(\sigma ; A)]^{\alpha}
$$

can be verified. Note that

$$
\left\|[I-(\sigma-\lambda) R(\sigma ; A)]^{\alpha}\right\| \leq \sum_{j=0}^{\infty}\left|\left(\begin{array}{l}
\alpha \\
j
\end{array}\right)\right| M=: C_{\alpha} .
$$


By the convolution theorem we obtain from (2) that for each $f \in X, R^{\alpha}(\sigma ; A) f \in$ $D\left((-A)^{\alpha}\right)$ and

$$
(\lambda I-A)^{\alpha} R^{\alpha}(\sigma ; A) f=[I-(\sigma-\lambda) R(\sigma ; A)]^{\alpha} f, \quad \sigma>0,0 \leq \lambda \leq \sigma .
$$

In particular, if we replace $f \in X$ by $R^{\alpha}(\lambda ; A) f$ we have for each $f \in X$

$$
R^{\alpha}(\sigma ; A) f=[I-(\sigma-\lambda) R(\sigma ; A)]^{\alpha} R^{\alpha}(\lambda ; A) f, \quad 0<\lambda \leq \sigma .
$$

For later reference we note

$$
\begin{aligned}
{[I-\sigma R(\sigma ; A)]^{\alpha} f } & =(-A)^{\alpha} R^{\alpha}(\sigma ; A) f, \quad f \in X, 0<\lambda \leq \sigma, \\
& =[I-(\sigma-\lambda) R(\sigma ; A)]^{\alpha}[I-\lambda R(\lambda ; A)]^{\alpha} f .
\end{aligned}
$$

\section{Proofs}

3.1. Proof of Theorem 1.4. For all $g \in D\left((-A)^{\alpha}\right)$ we have

$$
\begin{aligned}
\left\|[I-\lambda R(\lambda ; A)]^{\alpha} f\right\| & \leq\left\|[I-\lambda R(\lambda ; A)]^{\alpha}(f-g)\right\|+\left\|[I-\lambda R(\lambda ; A)]^{\alpha} g\right\| \\
& \leq C_{\alpha}\|f-g\|+\left\|R^{\alpha}(\lambda ; A)(-A)^{\alpha} g\right\| \\
& \leq c\left\{\|f-g\|+\lambda^{-\alpha}\left\|(-A)^{\alpha} g\right\|\right\} .
\end{aligned}
$$

Taking the infimum with respect to $g \in D\left((-A)^{\alpha}\right)$ yields

$$
\left\|[I-\lambda R(\lambda ; A)]^{\alpha} f\right\| \leq c K\left(\lambda^{-\alpha}, f ; X, D\left((-A)^{\alpha}\right)\right) .
$$

For the converse we consider the following family of operators $\left\{Z_{\lambda}: \lambda>0\right\}:$ Let $r \in \mathbf{N}$ be such that $r-1<\alpha \leq r$ and set

$$
Z_{\lambda}:=\sum_{k=1}^{\infty}(-1)^{k}\left(\begin{array}{l}
\alpha \\
k
\end{array}\right) \sum_{j=1}^{r}(-1)^{j}\left(\begin{array}{l}
r \\
j
\end{array}\right) j^{k} \lambda^{k} R^{k}(j \lambda ; A) .
$$

Certainly, this family is uniformly bounded with respect to $\lambda$ and

$$
I-Z_{\lambda}=\sum_{j=1}^{r}(-1)^{j-1}\left(\begin{array}{l}
r \\
j
\end{array}\right)[I-j \lambda R(j \lambda ; A)]^{\alpha} .
$$

Now observe, by (6) with $\sigma=j \lambda$, that

$$
[I-j \lambda R(j \lambda ; A)]^{\alpha}=[I-(j-1) \lambda R(j \lambda ; A)]^{\alpha}[I-\lambda R(\lambda ; A)]^{\alpha} .
$$

Therefore,

$$
I-Z_{\lambda}=\sum_{j=1}^{r}(-1)^{j-1}\left(\begin{array}{l}
r \\
j
\end{array}\right)[I-(j-1) \lambda R(j \lambda ; A)]^{\alpha}[I-\lambda R(\lambda ; A)]^{\alpha}
$$

and hence by (3), for all $f \in X$,

$$
\left\|\left[I-Z_{\lambda}\right] f\right\| \leq C_{\alpha} \sum_{j=1}^{r}\left(\begin{array}{l}
r \\
j
\end{array}\right)\left\|[I-\lambda R(\lambda ; A)]^{\alpha} f\right\| .
$$

It remains to show that $Z_{\lambda} f \in D\left((-A)^{\alpha}\right)$ for all $f \in X$ and

$$
\lambda^{-\alpha}\left\|(-A)^{\alpha} Z_{\lambda} f\right\| \leq c\left\|[I-\lambda R(\lambda ; A)]^{\alpha} f\right\| .
$$


To this end we split the infinite sum in the definition of $Z_{\lambda}$ into two parts:

$$
Z_{\lambda}=\left(\sum_{k=1}^{r-1}+\sum_{k=r}^{\infty}\right)(-1)^{k}\left(\begin{array}{l}
\alpha \\
k
\end{array}\right) \sum_{j=1}^{r}(-1)^{j}\left(\begin{array}{l}
r \\
j
\end{array}\right) j^{k} \lambda^{k} R^{k}(j \lambda ; A)=: S_{1}+S_{2} .
$$

Let us first discuss $S_{2}$ : For all $k \geq r$ we have by (1) and (6)

$$
\begin{aligned}
(-A)^{\alpha} R^{k}(j \lambda ; A) & =R^{k-\alpha}(j \lambda ; A)(-A)^{\alpha} R^{\alpha}(j \lambda ; A) \\
& =R^{k-\alpha}(j \lambda ; A)[I-(j-1) \lambda R(j \lambda ; A)]^{\alpha}[I-\lambda R(\lambda ; A)]^{\alpha} .
\end{aligned}
$$

Hence, $S_{2} f \in D\left((-A)^{\alpha}\right)$ for all $f \in X$ and

$$
\begin{aligned}
(-A)^{\alpha} S_{2} f=\lambda^{\alpha} \sum_{k=r}^{\infty}(-1)^{k} & \left(\begin{array}{l}
\alpha \\
k
\end{array}\right) \sum_{j=1}^{r}(-1)^{j}\left(\begin{array}{l}
r \\
j
\end{array}\right) j^{\alpha}(j \lambda)^{k-\alpha} R^{k-\alpha}(j \lambda ; A) \\
& \times[I-(j-1) \lambda R(j \lambda ; A)]^{\alpha}[I-\lambda R(\lambda ; A)]^{\alpha} f
\end{aligned}
$$

and thus, for all $f \in X$,

$$
\lambda^{-\alpha}\left\|(-A)^{\alpha} S_{2} f\right\| \leq c\left\|[I-\lambda R(\lambda ; A)]^{\alpha} f\right\| .
$$

Let us now discuss the contribution coming from $S_{1}$ : For all $\lambda>0$ we have that $S_{1}$ is a linear combination of the linear operators $U_{\lambda, k}, k=1, \ldots, r-1$,

$$
U_{\lambda, k}:=\sum_{j=1}^{r}(-1)^{j}\left(\begin{array}{l}
r \\
j
\end{array}\right) j^{k} \lambda^{k} R^{k}(j \lambda ; A) .
$$

We claim that $U_{\lambda, k}$ can be represented in the form

$$
U_{\lambda, k}=H_{\lambda, k} \lambda^{r} R^{r}(\lambda ; A),
$$

where

$$
\begin{aligned}
H_{\lambda, k}= & r !\left(\sum_{m=k+1}^{r}(-1)^{m}\left(\begin{array}{c}
r \\
m
\end{array}\right)\right) I \\
& +\sum_{m=0}^{k}\left(\begin{array}{c}
r \\
m
\end{array}\right) \sum_{j=1}^{r}(-1)^{j}\left(\begin{array}{l}
r \\
j
\end{array}\right) j^{k}(1-j)^{r-m} \lambda^{k-m} R^{k-m}(j \lambda ; A) .
\end{aligned}
$$

Clearly, for $k=1, \ldots, r-1$, we have that $\left\{H_{\lambda, k}: \lambda>0\right\}$ is a uniformly bounded family (with respect to $\lambda>0$ ) of linear operators. Further, by (1) and (4),

$$
\begin{aligned}
(-A)^{\alpha} U_{\lambda, k} & =\lambda^{\alpha} H_{\lambda, k} \lambda^{r-\alpha} R^{r-\alpha}(\lambda ; A)(-A)^{\alpha} R^{\alpha}(\lambda ; A) \\
& =\lambda^{\alpha} H_{\lambda, k} \lambda^{r-\alpha} R^{r-\alpha}(\lambda ; A)[I-\lambda R(\lambda ; A)]^{\alpha} .
\end{aligned}
$$

Hence, $S_{1} f \in D\left((-A)^{\alpha}\right)$ for all $f \in X$ and

$$
\begin{aligned}
(-A)^{\alpha} S_{1} f & =\sum_{k=1}^{r-1}(-1)^{k}\left(\begin{array}{l}
\alpha \\
k
\end{array}\right)(-A)^{\alpha} U_{\lambda, k} f \\
& =\lambda^{\alpha} \sum_{k=1}^{r-1}(-1)^{k}\left(\begin{array}{l}
\alpha \\
k
\end{array}\right) H_{\lambda, k} \lambda^{r-\alpha} R^{r-\alpha}(\lambda ; A)[I-\lambda R(\lambda ; A)]^{\alpha} f .
\end{aligned}
$$


Taking norms we obtain for all $f \in X$

$$
\frac{1}{\lambda^{\alpha}}\left\|(-A)^{\alpha} S_{1} f\right\| \leq \sum_{k=1}^{r-1}\left|\left(\begin{array}{l}
\alpha \\
k
\end{array}\right)\right|\left\|H_{\lambda, k}\right\| M\left\|[I-\lambda R(\lambda ; A)]^{\alpha} f\right\| .
$$

This together with (8) proves (7), provided (9) is true, and the desired converse direction will follow:

$$
K\left(\frac{1}{\lambda^{\alpha}}, f ; X, D\left((-A)^{\alpha}\right)\right) \leq\left\|f-Z_{\lambda} f\right\|+\frac{1}{\lambda^{\alpha}}\left\|(-A)^{\alpha} Z_{\lambda} f\right\| \leq c\left\|[I-\lambda R(\lambda ; A)]^{\alpha} f\right\| .
$$

It remains to show that $(9)$ is true. First observe that it is enough to prove $(9)$ for $\lambda=1$. By (1) and (5) we have for $k=1, \ldots, r-1$

$$
R^{k}(j ; A)=(j I-A)^{r-k} R^{r}(j ; A)=(j I-A)^{r-k}[I-(j-1) R(j ; A)]^{r} R^{r}(1 ; A) .
$$

Thus,

$$
\begin{aligned}
U_{1, k} & =\left(\sum_{m=0}^{k}+\sum_{m=k+1}^{r}\right)\left(\begin{array}{c}
r \\
m
\end{array}\right) \sum_{j=1}^{r}(-1)^{j}\left(\begin{array}{l}
r \\
j
\end{array}\right) j^{k}(1-j)^{r-m}(j I-A)^{r-k} R^{r-m}(j ; A) R^{r}(1 ; A) \\
& =: Q_{1}+Q_{2} .
\end{aligned}
$$

Clearly, $Q_{1}$ coincides with the corresponding term in 97 for $\lambda=1$. Concerning $Q_{2}$ note that

$$
\begin{aligned}
& \sum_{j=1}^{r}(-1)^{j}\left(\begin{array}{l}
r \\
j
\end{array}\right) j^{k}(1-j)^{r-m}(j I-A)^{m-k} R^{r}(1 ; A) \\
& =\sum_{\rho=0}^{m-k}\left(\begin{array}{c}
m-k \\
\rho
\end{array}\right) \sum_{j=1}^{r}(-1)^{j}\left(\begin{array}{c}
r \\
j
\end{array}\right) j^{k+\rho}(1-j)^{r-m}(-A)^{m-k-\rho} R^{r}(1 ; A) \\
& =(-1)^{m} r ! R^{r}(1 ; A),
\end{aligned}
$$

since for $k=1, \ldots, r-1$ and $m=k+1, \ldots, r$ we have

$$
\sum_{j=1}^{r}(-1)^{j}\left(\begin{array}{l}
r \\
j
\end{array}\right) j^{k+\rho}(1-j)^{r-m}= \begin{cases}0, & \rho=0,1, \ldots, m-k-1, \\
(-1)^{m} r !, & \rho=m-k,\end{cases}
$$

cf., e.g. [2], p. 98. Therefore,

$$
Q_{2}=r !\left(\sum_{m=k+1}^{r}(-1)^{m}\left(\begin{array}{c}
r \\
m
\end{array}\right)\right) R^{r}(1 ; A),
$$

and the representation $\sqrt{9}$ is established; thus Theorem 1.4 is proved.

3.2. Proof of Theorem 1.2. Set $B_{\lambda}:=\left[I-\lambda R\left(\lambda ; A_{X}\right)\right]^{\alpha}, \lambda>\lambda_{0}$, and use the abbreviation $A:=A_{X}$. For all $h \in X$ we have $\lim _{\lambda \rightarrow \infty}\left\|B_{\lambda} h\right\|_{X}=0$. Since by (6)

$$
B_{2 \lambda}=[I-2 \lambda R(2 \lambda ; A)]^{\alpha}=[I-\lambda R(2 \lambda ; A)]^{\alpha}[I-\lambda R(\lambda ; A)]^{\alpha},
$$

we have

$$
B_{2 \lambda}-B_{\lambda}=\sum_{k=1}^{\infty}(-1)^{k}\left(\begin{array}{l}
\alpha \\
k
\end{array}\right) \lambda^{k} R^{k}(2 \lambda ; A)[I-\lambda R(\lambda ; A)]^{\alpha} .
$$


By the hypothesis $\left(\mathrm{N}_{\varphi, \lambda_{0}}^{*}\right)$ we have $B_{2 \lambda} f-B_{\lambda} f \in Y$ for each $f \in X$ and

$$
\begin{aligned}
\left\|B_{2 \lambda} f-B_{\lambda} f\right\|_{Y} & \leq \sum_{k=1}^{\infty}\left|\left(\begin{array}{c}
\alpha \\
k
\end{array}\right)\right| 2^{-k}\left\|(2 \lambda)^{k} R^{k}(2 \lambda ; A)[I-\lambda R(\lambda ; A)]^{\alpha} f\right\|_{Y} \\
& \leq c \varphi\left(\frac{1}{2 \lambda}\right) \sum_{k=1}^{\infty} 2^{-k}\left\|(2 \lambda)^{k-1} R^{k-1}(2 \lambda ; A)[I-\lambda R(\lambda ; A)]^{\alpha} f\right\|_{X} \\
& \leq c \varphi\left(\frac{1}{2 \lambda}\right) K\left(\lambda^{-\alpha}, f ; X, D\left((-A)^{\alpha}\right)\right)
\end{aligned}
$$

since the norm terms in the last sum can be estimated by

$$
\leq M\left\|[I-\lambda R(\lambda ; A)]^{\alpha} f\right\|_{X} \approx K\left(\lambda^{-\alpha}, f ; X, D\left((-A)^{\alpha}\right)\right) .
$$

If $1 /(4 \lambda)<u<1 /(2 \lambda)$, then $\varphi(1 /(2 \lambda)) \leq c \varphi(u)$ and $K\left(\lambda^{-\alpha}, f ; X, D\left((-A)^{\alpha}\right)\right) \leq$ $c K\left(u^{\alpha}, f ; X, D\left((-A)^{\alpha}\right)\right)$ and, therefore,

$$
\varphi\left(\frac{1}{2 \lambda}\right) K\left(\lambda^{-\alpha}, f ; X, D\left((-A)^{\alpha}\right)\right) \leq c \int_{1 /(4 \lambda)}^{1 /(2 \lambda)} \varphi(u) K\left(u^{\alpha}, f ; X, D\left((-A)^{\alpha}\right)\right) \frac{d u}{u}
$$

dominates $\left\|B_{2 \lambda} f-B_{\lambda} f\right\|_{Y}$. Now observe that for $m>k$

$$
\begin{aligned}
\left\|B_{2^{m} \lambda} f-B_{2^{k} \lambda} f\right\|_{Y} & \leq \sum_{\ell=k+1}^{m}\left\|\left[B_{2^{\ell} \lambda} f-B_{2^{\ell-1} \lambda} f\right]\right\|_{Y} \\
& \leq c \sum_{\ell=k+1}^{m} \int_{1 /\left(2^{\ell+1} \lambda\right)}^{1 /\left(2^{\ell} \lambda\right)} \varphi(u) K\left(u^{\alpha}, f ; X, D\left((-A)^{\alpha}\right)\right) \frac{d u}{u} \\
& =\int_{1 /\left(2^{m+1} \lambda\right)}^{1 /\left(2^{k+1} \lambda\right)} \varphi(u) K\left(u^{\alpha}, f ; X, D\left((-A)^{\alpha}\right)\right) \frac{d u}{u}
\end{aligned}
$$

which tends to 0 for $m, k \rightarrow \infty$. Since for $k=0$ we have

$$
\left\|B_{2^{m} \lambda} f-B_{\lambda} f\right\|_{Y} \leq c \int_{1 /\left(2^{m+1} \lambda\right)}^{1 /(2 \lambda)} \varphi(u) K\left(u^{\alpha}, f ; X, D\left((-A)^{\alpha}\right)\right) \frac{d u}{u},
$$

we see by the previous estimate that $\left(B_{2^{m}} \lambda f-B_{\lambda} f\right)_{m}$ is a Cauchy sequence in $Y$. Since $X$ and $Y$ are compatible, the limit has to be $-B_{\lambda} f$. Thus $B_{\lambda} f \in Y$ and

$$
\left\|B_{\lambda} f\right\|_{Y} \leq c \int_{0}^{1 / \lambda} \varphi(u) K\left(u^{\alpha}, f ; X, D\left((-A)^{\alpha}\right)\right) \frac{d u}{u} .
$$

Now $f=B_{\lambda} f-\sum_{k=1}^{\infty}(-1)^{k}\left(\begin{array}{l}\alpha \\ k\end{array}\right) \lambda^{k} R^{k}(\lambda ; A) f \in Y$. Thus, by Theorem 1.4 ,

$$
K\left(\lambda^{-\alpha}, f ; Y, D_{Y}\left((-A)^{\alpha}\right)\right) \approx\left\|B_{\lambda} f\right\|_{Y} \leq c \int_{0}^{1 / \lambda} \varphi(u) K\left(u^{\alpha}, f ; X, D\left((-A)^{\alpha}\right)\right) \frac{d u}{u},
$$

i.e., we have shown the assertion.

Acknowledgement. The deduction of the formula (9) has been technically revised at a referee's suggestion for which the authors are grateful. 


\section{References}

[1] R. A. Adams, Sobolev Spaces, Academic Press, New York, 1975.

[2] P. L. Butzer and H. Berens, Semi-Groups of Operators and Approximation, Springer, Berlin, 1967.

[3] A. Lunardi, Analytic Semigroups and Optimal Regularity in Parabolic Problems, Birkhäuser, Basel, 1995.

[4] C. Martinez and M. Sanz, The Theory of Fractional Powers of Operators, Elsevier, Amsterdam, 2001.

[5] J. Peetre, A Theory of Interpolation of Normed Spaces, Lecture Notes, Brasilia, 1963 [Notas de matematica 39 (1968), 1-86].

[6] L. Schwartz, Lectures on Mixed Problems in Partial Differential Equations and Representations of Semi-Groups, Tata Institute of Fundamental Research, Bombay, 1958.

[7] W. Trebels and U. Westphal, Characterizations of $K$-functionals built from fractional powers of infinitesimal generators of semigroups, Constr. Approx. 19 (2003), 355-371.

[8] W. Trebels and U. Westphal, On Ulyanov inequalities in Banach spaces and semigroups of linear operators, J. Approx. Theory 160 (2009), 154-170.

[9] U. Westphal, Fractional powers of infinitesimal generators of semigroups, in: Applications of Fractional Calculus in Physics, R. Hilfer (ed.), World Sci. Publ., River Edge, NJ, 2000, 131-170. 
